\title{
Applications of Categorical Fixed-basis Fuzzy Topological Spaces to A-valued Spaces
}

\author{
Mustafa Demirci ${ }^{1}$ \\ ${ }^{1}$ Akdeniz University, Faculty of Sciences, Department of Mathematics, 07058-Antalya, Turkey
}

\begin{abstract}
As an application of the dual equivalence between the category of $L$-spatial $\mathbf{C}$-objects and the category of $L$-sober $\mathbf{C}$ - $\mathcal{M}$ - $L$-spaces, it is shown in this paper that for a fixed augmented partially ordered set $A$, there exists a dual equivalence between the category of $A$-spatial augmented partially ordered sets and the category of $A$-sober $A$-valued spaces. Then, with regard to this duality, for a fixed $\left(\mathcal{Z}_{1}, \mathcal{Z}_{2}\right)$ complete partially ordered set $L$, we establish a dual equivalence between the category of $L$-spatial $\left(\mathcal{Z}_{1}, \mathcal{Z}_{2}\right)$-complete partially ordered sets and the category of $L$-sober $L$-valued $\mathcal{Q}$-spaces.
\end{abstract}

Keywords: Fuzzy topology, categorical fuzzy topology, partially ordered set-valued space, augmented partially ordered set, complete partially ordered set, subset system

\section{Introduction}

The famous adjunction $\Omega \dashv P t$ between the category Top of topological spaces and the opposite Loc of the category Frm of frames [16, 17, 19], known as Papert-Papert-Isbell adjunction [24], and its various generalizations in fuzzy set theory have received much attention during the last three decades $[2,3,4,5,14,17,20,23,24]$. Also see [4, 24] for many other references not included in this paper. An abstract categorical analogue of Papert-PapertIsbell adjunction, replacing Loc with the opposite $\mathbf{C}^{o p}$ of an abstract category $\mathbf{C}$, and Top with the category C- $\mathcal{M}-L$-Top of C- $\mathcal{M}-L$-spaces, is formulated to be the adjunction $L \Omega_{\mathcal{M}} \dashv L P t_{\mathcal{M}}: \mathbf{C}^{o p} \rightarrow$ C- $\mathcal{M}-L$-Top in [5]. As a natural categorical generalization of the famous equivalence between the full subcategory of Loc of all spatial locales and the full subcategory of Top of all sober topological spaces $[16,17]$, the adjunction between $\mathbf{C}-\mathcal{M}-L$-Top and $\mathbf{C}^{o p}$ is also refined to a dual equivalence between the full subcategory $L$-Spat-C of $\mathbf{C}$ of all $L$-spatial objects and the full subcategory C-M- $L$-SobTop of C-M- $L$-Top of all $L$-sober objects in [5].

Partially ordered set (poset for short) with the additional information of certain specified joins and meets has been a significant issue in order theory, algebra, computer science and topology, see $[4,7,8]$ and the references therein. There are two main approaches: The first one involves the notion of augmented poset, proposed by Banaschewski and
Bruns [2], while the second one relies on the notion of $\mathcal{Z}$-complete poset [7]. Augmented posets constitute a category $\mathbf{P}$. The adjunction $T \dashv \Psi$ : $\mathbf{P}^{o p} \rightarrow \mathbf{S}$ between the category $\mathbf{S}$ of spaces and the opposite $\mathbf{P}^{o p}$ of $\mathbf{P}$ and is one of the central results of [2], and the dual equivalence between the full subcategory $\mathbf{S p a P}$ of $\mathbf{P}$ of all spatial objects and the full subcategory SobS of $\mathbf{S}$ of all sober objects is another one. The second approach uses the notion of subset selection $[6,7]$, that is, a rule $\mathcal{Z}$ assigning to each partially ordered set (poset for short) $P$ a subset $\mathcal{Z}(P)$ of the power set $\mathcal{P}(P)$ of $P$, and is extended to the framework of $\left(\mathcal{Z}_{1}, \mathcal{Z}_{2}\right)$ complete posets in [4]. Here the subset selection generalizes the notion of subset system [6], originally introduced by Wright et. al. [25]. For a quadruple $\mathcal{Q}=\left(\mathcal{Z}_{1}, \mathcal{Z}_{2}, \mathcal{Z}_{3}, \mathcal{Z}_{4}\right)$ of subset systems $\mathcal{Z}_{1}, \mathcal{Z}_{2}, \mathcal{Z}_{3}$ and $\mathcal{Z}_{4},\left(\mathcal{Z}_{1}, \mathcal{Z}_{2}\right)$-complete posets and $\left(\mathcal{Z}_{3}, \mathcal{Z}_{4}\right)$-continuous functions form a category $\mathcal{Q P}$ [4]. $\mathcal{Q}$-spaces and their category $\mathcal{Q S}$ have been introduced in [4] as a topological counterpart of $\mathcal{Q P}$. Furthermore, by establishing two full embeddings $G_{\mathcal{Q}}: \mathcal{Q P} \rightarrow \mathbf{P}$ and $H_{\mathcal{Q}}: \mathcal{Q S} \rightarrow \mathbf{S}$, it is demonstrated in [4] that the first approach is categorytheoretically more general approach than the second one, while the latter yields feasible results in applications. Despite the fact that both approaches provide useful and powerful tools to unify various kinds of generalized topological spaces under the same framework, they are inadequate to handle the problems related with the poset-valued extensions of such generalized topological spaces resulting from the essence of fuzzy set theory. To overcome this shortcoming, we introduce poset-valued spaces and poset-valued $\mathcal{Q}$-spaces, and extend all central results of $[2,4]$ to the present approach. More specifically, referring to a fixed augmented poset $A$ and a fixed $\left(\mathcal{Z}_{1}, \mathcal{Z}_{2}\right)$-complete poset $L$, we extend $\mathbf{S}$ and $\mathcal{Q} \mathbf{S}$ to the category $A-\mathbf{S}$ of $A$-valued spaces and the category $L-\mathcal{Q S}$ of $L$-valued $\mathcal{Q}$-spaces, respectively. Then, we apply the adjunction $L \Omega_{\mathcal{M}} \dashv L P t_{\mathcal{M}}$ to augmented posets and $A$-valued spaces, and obtain the adjunction $A T \dashv A \Psi: \mathbf{P}^{o p} \rightarrow A-\mathbf{S}$ as a generalization of $T \dashv \Psi: \mathbf{P}^{o p} \rightarrow \mathbf{S}$ in Theorem 16. The dual equivalence between the full subcategory of $\mathbf{P}$ of all $A$-spatial augmented posets and the full subcategory of $A$-S of all $A$-sober $A$-spaces is proven to be an instance of the equivalence between ( $L$ Spat-C) ${ }^{o p}$ and C- $\mathcal{M}$ - $L$-SobTop in Corollary 18, and extends the equivalence between $\mathbf{S p a P}^{o p}$ and 


\section{SobS.}

The main result of [4] ([4, Theorem 2]) is reformulated for $L$-valued $\mathcal{Q}$-spaces in Theorem 31 consisting of two adjunctions between $L-\mathcal{Q S}$ and the opposite of the full subcategory $L-\mathcal{Q} \mathbf{P}_{s}$ of $\mathcal{Q P}$ of all $L$ - $\mathcal{Q}$-spatial objects, $L-\left(\mathcal{Z}_{1}, \mathcal{Z}_{2}, \mathcal{Z}_{1}, \mathcal{Z}_{2}\right) \mathbf{S}$ and the opposite of $\left(\mathcal{Z}_{1}, \mathcal{Z}_{2}, \mathcal{Z}_{1}, \mathcal{Z}_{2}\right) \mathbf{P}$ and a dual equivalence between $L-\mathcal{Q} \mathbf{P}_{s}$ and the full subcategory of $L-\mathcal{Q S}$ of all $L-\mathcal{Q}$-sober objects. In order to show the usefulness of the presented results, we give their direct applications to some familiar order-theoretic categories in Corollary 32.

\section{Categorical fixed-basis fuzzy topological spaces and their duality}

\subsection{The category-theoretic fixed-basis fuzzy topological spaces}

To introduce fixed-basis fuzzy topological spaces in an abstract category $\mathbf{C}$ and their category, we begin with necessary category-theoretic preliminaries, and refer to $[1,13,18]$ for all other notions, notations and facts from category theory. First of all, we assume $\mathbf{C}$ as an abstract category with products, and fix a $\mathbf{C}$-object $L$ and a class $\mathcal{M}$ of $\mathbf{C}$ monomorphisms. Whenever further assumptions on C, $L$ and $\mathcal{M}$ are necessary, they will be clearly mentioned. Given a set $X$, an $X$-th power of $L$ is a C-object $L^{X}$ such that $\left(L^{X} \stackrel{\pi_{x}}{\rightarrow} L_{x}\right)_{x \in X}$ is a product where $L_{x}=L$ for each $x \in X$. Each function $f: X \rightarrow Y$ determines a unique $\mathbf{C}$-morphism $f_{L}^{\leftarrow}: L^{Y} \rightarrow L^{X}$ (the so-called backward $\mathbf{C}-L$ powerset operator of $f$ ) such that the triangle

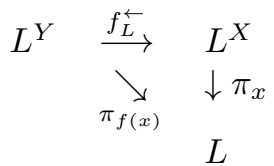

commutes for all $x \in X$. The notion of backward $\mathbf{C}$ $L$-powerset operator is a category-theoretic generalization of the $L$-powerset operator which has been extensively used in fuzzy (lattice-valued) mathematics [20, 21, 22, 23].

Proposition 1. Let $\boldsymbol{C}$ be a construct (i.e. a concrete category over the category Set of sets and functions with the forgetful functor ||$: \mathbf{C} \rightarrow$ Set) with the property that for each set $X$ and $x \in X$, $\left|L^{X}\right|=|L|^{X}$ and $\left|\pi_{x}\right|$ is the $x$-th projection map of $|L|^{X}$. Then, for any function $f: X \rightarrow Y$, the underlying function of $f_{L}^{\leftarrow}: L^{Y} \rightarrow L^{X}$ is given by the $\operatorname{map} f_{|L|}^{\leftarrow}:|L|^{Y} \rightarrow|L|^{X}, \mu \mapsto f_{|L|}^{\leftarrow}(\mu)=\mu \circ f$

Note that all constructs $\mathbf{C}$ in Example 3 below satisfy the hypothesis of Proposition 1 . For the particular case $\mathbf{C}=$ Set and $L=2, f_{2}^{\leftarrow}: 2^{Y} \rightarrow 2^{X}$ corresponds to the traditional backward (preimage) powerset operator $f^{\leftarrow}: \mathcal{P}(Y) \rightarrow \mathcal{P}(X)$ in the usual sense [21], where $\mathcal{P}(X)$ stands for the powerset of
$X$. However, neither the traditional forward (image) powerset operator $f \rightarrow: \mathcal{P}(X) \rightarrow \mathcal{P}(Y)$ nor its lattice-valued generalizations in $[20,21,22]$ have a category-theoretic counterpart in $\mathbf{C}$.

Definition 2. [5] C-M-L-Top is a category whose objects (the so-called $\mathbf{C}$ - $\mathcal{M}$-L-spaces) are pairs $\left(X, \tau \stackrel{m}{\rightarrow} L^{X}\right)$, consisting of a set $X$ and an $\mathcal{M}$-morphism $\tau \stackrel{m}{\rightarrow} L^{X}$, and whose morphisms $\left(X, \tau \stackrel{m_{1}}{\rightarrow} L^{X}\right) \stackrel{f}{\rightarrow}\left(Y, \nu \stackrel{m_{2}}{\rightarrow} L^{Y}\right) \quad$ (the so-called $\mathbf{C}$ $\mathcal{M}$-L-continuous maps) are functions $f: X \rightarrow Y$ such that there exists a (necessarily unique) $\mathbf{C}$ morphism $r_{f}: \nu \rightarrow \tau$ making the diagram

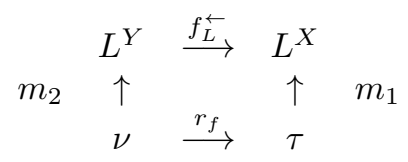

commutative.

C- $\mathcal{M}-L$-Top unifies most of the categorytheoretic approaches to the fixed-basis fuzzy topological spaces. To show its value and usefulness, we gathered some familiar cases of $\mathbf{C}-\mathcal{M}$ - $L$-Top in the following example.

Example 3. Along this example, $\boldsymbol{C}$ is assumed be a construct, while $\mathcal{M}_{\mathbf{C}}$ always denotes the class of all $\boldsymbol{C}$-morphisms $m: A \rightarrow B$ such that $|A| \subseteq|B|$ and $|m|:|A| \hookrightarrow|B|$ is the inclusion map.

(i) For $\boldsymbol{C}$ chosen as the category $\boldsymbol{C G R}$ of complete groupoids in [14], C-M $\mathcal{M}_{\mathbf{C}}-L-T o p$ is isomorphic to the category $L$-TOP of L-valued topological spaces in [14].

(ii) For $\boldsymbol{C}$ chosen as the category $\boldsymbol{C Q M L}$ of complete quasi-monoidal lattices in [15], C- $\mathcal{M}_{\mathbf{C}}-L$ Top is isomorphic to the category $L-\mathbf{T O P}$ of $L$ topological spaces in [15].

(iii) For $\boldsymbol{C}$ chosen as the category SQuant of semi-quantales in [22], C- $\mathcal{M}_{\mathbf{C}}-L-$ Top is isomorphic to the category L-QTop of L-quasi-topological spaces in [22].

(iv) For $\boldsymbol{C}$ chosen as the category USQuant of unital semi-quantales in [22], C- $\mathcal{M}_{\mathbf{C}}-L$-Top is isomorphic to the category L-Top of L-topological spaces in [22].

(v) For $\boldsymbol{C}$ chosen as a variety $\boldsymbol{V R}$ (i.e. a full subcategory of the category $\boldsymbol{A l g}(\Omega)$ of $\Omega$-algebras and $\Omega$-homomorphisms closed under the formation of products, subalgebras and homomorphic images [23]), C-M $\mathcal{M}_{\mathbf{C}}-L-$ Top is isomorphic to the category $L$-Top of L-topological spaces in [23]. In particular, if $\boldsymbol{C}$ is taken as the variety $\mathbf{S F r m}$ [22] of semiframes or the variety $\mathbf{F r m}$ [17] of frames, then $\mathbf{C}$ $\mathcal{M}_{\mathbf{C}-2-T o p}$ is isomorphic to the category Top of topological spaces in the usual sense.

\subsection{An adjunction between C- $\mathcal{M}-L$-Top and $\mathrm{C}^{o p}$}

C is called an essentially $(\mathcal{E}, \mathcal{M})$-structured category iff $(\mathcal{E}, \mathcal{M})$ is a pair of $\mathbf{C}$-morphisms classes 
satisfying the following conditions [5]:

- $\mathbf{C}$ has $(\mathcal{E}, \mathcal{M})$-factorizations, i.e. each $\mathbf{C}$ morphism $f$ has an $(\mathcal{E}, \mathcal{M})$-factorization pair $(e, m)$, i.e. $f=m \circ e$ with $e \in \mathcal{E}$ and $m \in \mathcal{M}$.

- $\mathbf{C}$ has the unique $(\mathcal{E}, \mathcal{M})$-diagonalization property, i.e. for any $e \in \mathcal{E}, m \in \mathcal{M}$ and $f, g \in$ $\operatorname{Mor}(\mathbf{C})$ such that $g \circ e=m \circ f$, there exists a unique C-morphism $d$ fulfilling $d \circ e=f$ and $m \circ d=g$.

The only difference between an essentially $(\mathcal{E}, \mathcal{M})$-structured category and an $(\mathcal{E}, \mathcal{M})$ structured category in $[1,13]$ is the lack of the closedness of both $\mathcal{E}$ and $\mathcal{M}$ under the composition with isomorphisms in the former. If we reconsider Example 3, then for the class $\operatorname{Surj}(\mathbf{C})$ of C-morphisms with surjective underlying functions, all constructs $\mathbf{C}$ in Example 3 are essentially $\left(\operatorname{Surj}(\mathbf{C}), \mathcal{M}_{\mathbf{C}}\right)$-structured. But, since $\mathcal{M}_{\mathbf{C}}$ is not closed under the composition with isomorphisms, non of them are $\left(\operatorname{Surj}(\mathbf{C}), \mathcal{M}_{\mathbf{C}}\right)$-structured!

Let us recall that an adjoint situation $(\eta, \varepsilon): F \dashv$ $G: \mathbf{A} \rightarrow \mathbf{B}$ is a pair of functors $G: \mathbf{A} \rightarrow \mathbf{B}$ and $F: \mathbf{B} \rightarrow \mathbf{A}$ with the unit $i d_{\mathbf{B}} \stackrel{\eta}{\rightarrow} G \circ F$ and co-unit $F \circ G \stackrel{\varepsilon}{\rightarrow} i d_{\mathbf{A}}$ satisfying the adjunction identities: $G\left(\varepsilon_{A}\right) \circ \eta_{G(A)}=i d_{G(A)}$ and $\varepsilon_{F(B)} \circ F\left(\eta_{B}\right)=i d_{F(B)}$ for all $A$ in $\mathbf{A}$ and $B$ in B. $F$ is left adjoint to $G$, $F \dashv G$ in symbols, iff $(\eta, \varepsilon): F \dashv G: \mathbf{A} \rightarrow \mathbf{B}$ is an adjoint situation for some $\eta$ and $\varepsilon$. Two categories $\mathbf{A}$ and $\mathbf{B}$ are equivalent, $\mathbf{A} \sim \mathbf{B}$ in symbols, iff there exists an adjoint situation $(\eta, \varepsilon): F \dashv G: \mathbf{A} \rightarrow \mathbf{B}$ with natural isomorphisms $\eta$ and $\varepsilon$. Furthermore, we use the notation $\mathbf{A} \cong \mathbf{B}$ whenever $\mathbf{A}$ and $\mathbf{B}$ are isomorphic categories.

Proposition 4. [5] The class-theoretic function $L \Omega_{\mathcal{M}}: \boldsymbol{C}-\mathcal{M}-L-\boldsymbol{T o p} \rightarrow \boldsymbol{C}^{o p}$, defined by

$$
L \Omega_{\mathcal{M}}\left(\left(X, \tau \stackrel{m_{1}}{\rightarrow} L^{X}\right) \stackrel{f}{\rightarrow}\left(Y, \nu \stackrel{m_{2}}{\rightarrow} L^{Y}\right)\right)=\tau \stackrel{r_{f}^{o p}}{\rightarrow} \nu
$$

is a functor.

Each C-object $A$ determines a unique Cmorphism $\langle A\rangle: A \rightarrow L^{\mathbf{C}(A, L)}$ satisfying the property that $A \stackrel{h}{\rightarrow} L=A \stackrel{\langle A\rangle}{\rightarrow} L \mathbf{C}(A, L) \stackrel{\pi_{h}}{\rightarrow} L$ for each $h \in \mathbf{C}(A, L)$, where $\mathbf{C}(A, L)$ denotes the hom-set from $A$ to $L$. In the rest of this section, we assume that $\mathbf{C}$ is essentially $(\mathcal{E}, \mathcal{M})$-structured, and fix an $(\mathcal{E}, \mathcal{M})$-factorization $A \stackrel{e_{A}}{\rightarrow} \tau_{A} \stackrel{m_{A}}{\rightarrow} L^{\mathbf{C}(A, L)}$ of $\langle A\rangle: A \rightarrow L^{\mathbf{C}(A, L)}$ for each $\mathbf{C}$-object $A$.

Proposition 5. [5] The class-theoretic function $\mathrm{LPt}_{\mathcal{M}}: \boldsymbol{C}^{o p} \rightarrow \boldsymbol{C}-\mathcal{M}-L-\boldsymbol{T o p}$, defined by

$$
\begin{aligned}
\operatorname{LPt}_{\mathcal{M}}(A) & =\left(\mathbf{C}(A, L), \tau_{A} \stackrel{m_{A}}{\rightarrow} L^{\mathbf{C}(A, L)}\right) \text { and } \\
\operatorname{LPt}_{\mathcal{M}}(g)(u) & =u \circ g^{o p}
\end{aligned}
$$

for each $A \in O b(\mathbf{C}), A_{1} \stackrel{g}{\rightarrow} A_{2} \in \operatorname{Mor}\left(\mathbf{C}^{o p}\right)$ and $u \in \mathbf{C}\left(A_{1}, L\right)$, is a functor.
Proposition 6. [5] (i) For each C-M-L-Topobject $W=\left(X, \tau \stackrel{m}{\rightarrow} L^{X}\right)$, the map $\eta_{W}: X \rightarrow$ $\mathbf{C}(\tau, L)$, defined by $\eta_{W}(x)=\pi_{x} \circ m$, is a $\mathbf{C}-\mathcal{M}$ $L$-Top-morphism $W \stackrel{\eta_{W}}{\rightarrow} \operatorname{LPt}_{\mathcal{M}}\left(L \Omega_{\mathcal{M}}(W)\right)$.

(ii) For each $\mathbf{C}$-object $A$, the arrow $\varepsilon_{A}=e_{A}^{o p}$ : $\tau_{A} \rightarrow A$ is a $\mathbf{C}^{o p}$-morphism $L \Omega_{\mathcal{M}}\left(\operatorname{LPt}_{\mathcal{M}}(A)\right) \rightarrow$ $A$.

Theorem 7. [5] Let $\eta=\left(\eta_{W}\right)_{W \in O b(\mathbf{C}-\mathcal{M}-L-\mathbf{T o p})}$ and $\varepsilon=\left(\varepsilon_{A}\right)_{A \in O b(\mathbf{C})}$. Then $(\eta, \varepsilon): L \Omega_{\mathcal{M}} \dashv L P t_{\mathcal{M}}$ : $\mathbf{C}^{o p} \rightarrow$ C-M-L-Top.

Definition 8. (i) $A$ C-object $A$ is L-spatial iff for some $(\mathcal{E}, \mathcal{M})$-factorization pair $(e, m)$ of $A \stackrel{\langle A\rangle}{\rightarrow}$ $L^{\mathbf{C}(A, L)}$, e is an isomorphism in $\mathbf{C}$.

(ii) A C-M-L-Top-object $W=\left(X, \tau \stackrel{m}{\rightarrow} L^{X}\right)$ is $L$-sober iff for all $h \in \mathbf{C}(\tau, L)$, there exists a unique $x \in X$ such that $h=\pi_{x} \circ \mathrm{m}$.

Proposition 9. [5] (i) A C-object $A$ is L-spatial iff $\varepsilon_{A}^{o p}$ is an isomorphism in $\mathbf{C}$.

(ii) A C-M-L-Top-object $W$ is L-sober iff $\eta_{W}$ is an isomorphism in $\mathbf{C}-\mathcal{M}-L-\boldsymbol{T o p}$.

Corollary 10. [5] The full subcategory L-Spat-C of $\boldsymbol{C}$ of all L-spatial objects is dually equivalent to the full subcategory $\boldsymbol{C}-\mathcal{M}-L-S o b T o p$ of $\boldsymbol{C}-\mathcal{M}-L$ Top of all L-sober objects, i.e. (L-Spat-C $)^{o p} \sim$ C-M-L-SobTop.

\section{Applications to augmented posets}

\subsection{Category of augmented posets}

An augmented poset is a triple $A=(|A|, \mathfrak{J} A, \mathfrak{M} A)$, consisting of a poset $|A|$, a subset $\mathfrak{J} A$ of $\mathcal{P}(|A|)$ in which each member has the join in $|A|$ and a subset $\mathfrak{M} A$ of $\mathcal{P}(|A|)$ in which each member has the meet in $|A|$. Augmented posets together with structure preserving maps constitute a category $\mathbf{P}$ [2]. A structure preserving map $h: A \rightarrow B$ here means a monotone map $h:|A| \rightarrow|B|$ such that $h(S) \in \mathfrak{J} B$ and $h(\bigvee S)=\bigvee h(S)$ for all $S \in \mathfrak{J} A$, and $h(R) \in \mathfrak{M} B$ and $h(\bigwedge R)=\bigwedge h(R)$ for all $R \in \mathfrak{M} A[2] . \quad \mathbf{P}$ has products. In particular, for each set $X$ and an augmented poset $A$, the $X$-th power of $A$ is the augmented poset $A^{X}$ such that $\left|A^{X}\right|=|A|^{X}, \mathfrak{J} A^{X}$ is the set of subsets $S \subseteq|A|^{X}$ with the property that $\pi_{x}(S) \in \mathfrak{J} A$ for all $x \in X$, and analogously for $\mathfrak{M} A^{X}$. Due to the terminology of Goguen [12], the elements of $|A|^{X}$ are called $|A|$ sets, generalizing the fuzzy sets [26] to the case that the truth-value structure is an augmented poset.

\subsection{Category of poset-valued spaces and its duality}

$W=(|W|, \mathfrak{O}(W), \Sigma(W), \Delta(W))$ is called a space if $|W|$ is a set, $\mathfrak{O}(W)$ is a subset of $\mathcal{P}(|W|)$, $\Sigma(W)$ is a subset of $\{\mathfrak{U} \subseteq \mathfrak{O}(W) \mid \bigcup \mathfrak{U} \in \mathfrak{O}(W)\}$ and $\Delta(W)$ is a subset of $\{\mathfrak{V} \subseteq \mathfrak{O}(W) \mid \cap \mathfrak{V} \in \mathfrak{O}(W)\}$. 
Spaces form a category $\mathbf{S}[2]$ whose morphisms $f: W_{1} \rightarrow W_{2}$ are functions $f:\left|W_{1}\right| \rightarrow\left|W_{2}\right|$ satisfying the conditions that $\left(f^{\leftarrow}\right) \rightarrow\left(\mathfrak{O}\left(W_{2}\right)\right) \subseteq \mathfrak{O}\left(W_{1}\right)$, $\left(f^{\leftarrow}\right) \rightarrow(\mathfrak{U}) \in \Sigma\left(W_{1}\right)$ for each $\mathfrak{U} \in \Sigma\left(W_{2}\right)$, and $\left(f^{\leftarrow}\right) \rightarrow(\mathfrak{V}) \in \Delta\left(W_{1}\right)$ for each $\mathfrak{V} \in \Delta\left(W_{2}\right)$.

In the following considerations, we fix an augmented poset $A$, and extend the category of spaces to the category of $A$-valued spaces. Note first that for a function $f:\left|Z_{1}\right| \rightarrow\left|Z_{2}\right|$, by Proposition 1 , the backward $A$-powerset operator $f_{A}^{\leftarrow}: A^{\left|Z_{2}\right|} \rightarrow A^{\left|Z_{1}\right|}$ of $f$ is given by $f_{A}^{\leftarrow}(\mu)=\mu \circ f$ for each $\mu \in|A|^{\left|Z_{2}\right|}$.

Definition 11. The category A-S consists of the following information: Objects are A-valued spaces (A-spaces for short), i.e. quadruples $Z=(|Z|$, $\left.\mathfrak{O}_{A}(Z), \Sigma_{A}(Z), \Delta_{A}(Z)\right)$, where $|Z|$ is a set, $\mathfrak{O}_{A}(Z)$ is a subset of $|A|^{|Z|}, \Sigma_{A}(Z)$ is a set of subsets $\mathfrak{U} \subseteq \mathfrak{O}_{A}(Z)$ such that $\mathfrak{U} \in \mathfrak{J} A^{|Z|}$ and $\bigvee \mathfrak{U} \in \mathfrak{O}_{A}(Z)$, and $\Delta_{A}(Z)$ is a set of subsets $\mathfrak{V} \subseteq \mathfrak{O}_{A}(Z)$ such that $\mathfrak{V} \in \mathfrak{M} A^{|Z|}$ and $\bigwedge \mathfrak{V} \in \mathfrak{O}_{A}(Z)$, while morphisms $f: Z_{1} \rightarrow Z_{2}$ are functions $f:\left|Z_{1}\right| \rightarrow\left|Z_{2}\right|$ fulfilling the next properties: $\left(f_{A}^{\leftarrow}\right) \rightarrow\left(\mathfrak{O}_{A}\left(Z_{2}\right)\right) \subseteq \mathfrak{O}_{A}\left(Z_{1}\right)$, $\left(f_{A}^{\leftarrow}\right) \rightarrow(\mathfrak{U}) \in \Sigma_{A}\left(Z_{1}\right)$ for each $\mathfrak{U} \in \Sigma_{A}\left(Z_{2}\right)$, and $\left(f_{A}^{\leftarrow}\right) \rightarrow(\mathfrak{V}) \in \Delta_{A}\left(Z_{1}\right)$ for each $\mathfrak{V} \in \Delta_{A}\left(Z_{2}\right)$.

Remark 12. The category A-S can also be equivalently defined by means of the category $\boldsymbol{P}$ of augmented posets as follows.

(i) Let $Z=\left(|Z|, \mathfrak{O}_{A}(Z), \Sigma_{A}(Z), \Delta_{A}(Z)\right)$ be an entity consisting of a set $|Z|, \quad a$ subset $\mathfrak{O}_{A}(Z)$ of $|A|^{|Z|}$, subsets $\Sigma_{A}(Z)$ and $\Delta_{A}(Z)$ of $\mathcal{P}\left(|A|^{|Z|}\right)$. Then $Z$ is an $A$-space iff $A T(Z)=$ $\left(\mathfrak{O}_{A}(Z), \Sigma_{A}(Z), \Delta_{A}(Z)\right)$ is an augmented poset and the inclusion map $i_{A T(Z)}: A T(Z) \hookrightarrow A^{|Z|}$ is a $\boldsymbol{P}$ morphism.

(ii) For two $A$-spaces $Z_{1}, Z_{2}$ and a map $f$ : $\left|Z_{1}\right| \rightarrow\left|Z_{2}\right|, f: Z_{1} \rightarrow Z_{2}$ is an A-S-morphism iff the restriction of the $\boldsymbol{P}$-morphism $f_{A}^{\leftarrow}: A^{\left|Z_{2}\right|} \rightarrow$ $A^{\left|Z_{1}\right|}$ to $A T\left(Z_{2}\right)$ yields a $\boldsymbol{P}$-morphism $\left(f_{A}^{\leftarrow}\right)_{\left.\right|_{A T\left(Z_{2}\right)}}$ : $A T\left(Z_{2}\right) \rightarrow A T\left(Z_{1}\right)$.

We directly conclude from Remark 12:

Proposition 13. P-M $\mathcal{M}_{\mathbf{P}}-A-T o p \cong A-S$.

Proposition 14. For $2_{\mathbf{P}}=(2, \mathcal{P}(2), \mathcal{P}(2)), 2_{\mathbf{P}}-\boldsymbol{S}$ $\cong S$.

Proof. Given a set $X, \mathfrak{U} \subseteq \mathcal{P}(X)$ and $\Phi \subseteq$ $\mathcal{P}(\mathcal{P}(X))$, let $\mathfrak{U}^{c}$ denote the set of characteristic functions $\chi_{V}: X \rightarrow 2$ of all $V \in \mathfrak{U}$, and $\Phi^{*}=\left\{\mathfrak{U}^{c} \mid \mathfrak{U} \in \Phi\right\}$. Then, the functor $F: \mathbf{S} \rightarrow 2_{\mathbf{P}^{-}} \mathbf{S}$, defined by $F(W)=\left(|W|, \mathfrak{O}(W)^{c}, \Sigma(W)^{*}, \Delta(W)^{*}\right)$ for each $W \in O b(\mathbf{S})$, and $F(f)=f$ for each $f \in$ $\operatorname{Mor}(\mathbf{S})$, is an isomorphism proving the claim.

Corollary 15. $P-\mathcal{M}_{\mathrm{P}}-2_{\mathrm{P}}-T o p \cong S$.

Theorem 16. There exists an adjoint situation $\left(\eta^{A}, \varepsilon^{A}\right): A T \dashv A \Psi: \boldsymbol{P}^{o p} \rightarrow A-\boldsymbol{S}$.

Proof. Let us first show that $\mathbf{P}$ is essentially $\left(\right.$ ExtrEpi, $\left.\mathcal{M}_{\mathbf{P}}\right)$-structured. Let $h: U \rightarrow V$ be a $\mathbf{P}$-morphism. If we define the augmented poset Imh such that $|\operatorname{Imh}|$ is the set $h \rightarrow(U)$ equipped with the partial order inherited from $V, \mathfrak{J} \operatorname{Imh}=\{h \rightarrow(S) \mid S \in \mathfrak{J} U\}$ and $\mathfrak{M} \operatorname{Imh}=$ $\{h \rightarrow(S) \mid S \in \mathfrak{M} U\}$, then the inclusion map $i_{\text {Imh }}$ : $\operatorname{Imh} \hookrightarrow V$ is a $\mathbf{P}$-morphism. It is not difficult to see that the co-domain restriction of $h$ to $h \rightarrow(U)$ is an extremal epimorphism $e_{h}: U \rightarrow \operatorname{Imh}$ in $\mathbf{P}$. Thus, $U \stackrel{e_{h}}{\rightarrow} \operatorname{Imh} \stackrel{i_{\text {Imh }}}{\longrightarrow} V$ is an $\left(\right.$ ExtrEpi, $\left.\mathcal{M}_{\mathbf{P}}\right)$ factorization of $h: U \rightarrow V$. This means that $\mathbf{P}$ has $\left(\right.$ ExtrEpi, $\left.\mathcal{M}_{\mathbf{P}}\right)$-factorizations. On the other hand, since $\mathbf{P}$ has pullbacks [2], and by virtue of [13, 35.4 COROLLARY], $\mathbf{P}$ has the unique (ExtrEpi,Mono)-diagonalization property. Therefore, since $\mathcal{M}_{\mathbf{P}} \subseteq$ Mono, $\mathbf{P}$ has the unique $\left(\right.$ ExtrEpi, $\left.\mathcal{M}_{\mathbf{P}}\right)$-diagonalization property. Hence, $\mathbf{P}$ is essentially $\left(\operatorname{ExtrEpi}, \mathcal{M}_{\mathbf{P}}\right)$-structured. Then, since the hypothesis of Theorem 7 is satisfied for $\mathbf{C}=\mathbf{P}, \mathcal{E}=$ ExtrEpi and $\mathcal{M}=\mathcal{M}_{\mathbf{P}}$, the adjoint situation in question follows from Theorem 7 and Proposition 13.

By making use of the definitions of $\eta, \varepsilon, L \Omega_{\mathcal{M}}$ and $L P t_{\mathcal{M}}$, and by considering Proposition 13, one can calculate $\eta^{A}, \varepsilon^{A}, A T$ and $A \Psi$, explicitly. The functor $A T: A-\mathbf{S} \rightarrow \mathbf{P}^{o p}$ is given by $A T\left(Z_{1} \stackrel{f}{\longrightarrow} Z_{1}\right)=A T\left(Z_{1}\right) \stackrel{\left(f_{A}^{\leftarrow}\right)_{\left.\right|_{A T}}^{o p}}{\longrightarrow} A T\left(Z_{2}\right)$. To clarify the functor $A \Psi: \mathbf{P}^{o p} \rightarrow A-\mathbf{S}$, we first define, for each augmented poset $B$, the $A$-space $A \Psi(B)$ by $|A \Psi(B)|=\mathbf{P}(B, A), \mathfrak{O}_{A}(A \Psi(B))=\left\{\Psi_{a}|a \in| B \mid\right\}$, $\Sigma_{A}(A \Psi(B))=\left\{\left\{\Psi_{a} \mid a \in S\right\} \mid S \in \mathfrak{J} B\right\}$ and $\Delta_{A}(A \Psi(B))=\left\{\left\{\Psi_{a} \mid a \in S\right\} \mid S \in \mathfrak{M} B\right\}$, where $\Psi_{a}: \mathbf{P}(B, A) \rightarrow|A|$ is a map defined by $\Psi_{a}(h)=h(a)$ for each $a \in|B|$ and $h \in \mathbf{P}(B, A)$. Then, $A \Psi$ is given by

$$
A \Psi\left(B_{1} \stackrel{u}{\longrightarrow} B_{2}\right)=A \Psi\left(B_{1}\right) \stackrel{A \Psi(u)}{\longrightarrow} A \Psi\left(B_{2}\right),
$$

where $[A \Psi(u)](h)=h \circ u^{o p}$ for all $h \in\left|A \Psi\left(B_{1}\right)\right|$. Finally, for each $A$-space $W$ and for each augmented poset $B$, the $W$-th component of $\eta^{A}$ is the $A$-S-morphism $\eta_{W}^{A}: W \rightarrow A \Psi(A T(W))$, defined by $\left[\eta_{W}(x)\right](h)=h(x)$ for all $x \in|W|$ and $h \in|A T(W)|$, whereas the $B$-th component of $\varepsilon^{A}$ is the opposite of the $\mathbf{P}$-morphism $\left(\varepsilon_{B}^{A}\right)^{o p}: B \rightarrow$ $A T(A \Psi(B))$, defined by $\left(\varepsilon_{B}^{A}\right)^{o p}(a)=\Psi_{a}$ for each $a \in|B|$.

Definition 17. (i) An augmented poset $B$ is $A$ spatial iff $\left(\varepsilon_{B}^{A}\right)^{o p}$ is an isomorphism in $\boldsymbol{P}$.

(ii) An A-space $W$ is A-sober iff $\eta_{W}^{A}$ is an isomorphism in $A-S$.

Corollary 18. Let A-SpaP be the full subcategory of $\boldsymbol{P}$ of all A-spatial augmented posets, and A-SobS be the full subcategory of $A$ - $S$ of all $A$-sober $A$ spaces. Then, A-SpaP ${ }^{o p} \sim A-S o b S$.

Proof. Since $A$-SpaP $=A$-Spat-P and $A$-SobS $\cong$ $\mathbf{P}-\mathcal{M}_{\mathbf{P}}-A$-SobTop, the required equivalence follows from Corollary 10. 
Without going into detail, it is worth to mention here that $2 \mathbf{P}$-SpaP is the same as the full subcategory SpaP of $\mathbf{P}$ of all spatial augmented posets in [2], whereas $2 \mathbf{P}^{-} \mathbf{S o b S}$ is isomorphic to the full subcategory SobS of $\mathbf{S}$ of all sober spaces in [2]. Thus, the dual equivalence between $\mathbf{S p a P}$ and SobS proven in [2, Proposition 2] is an instance of Corollary 18. We close this section with the final remark that the co-domain of $A T$ lies in $A$-SpaP ${ }^{o p}$, while the co-domain of $A \Psi$ lies in $A$-SobS.

\section{Applications to $\left(\mathcal{Z}_{1}, \mathcal{Z}_{2}\right)$-complete posets}

\section{1. $\left(\mathcal{Z}_{1}, \mathcal{Z}_{2}\right)$-complete posets and their category}

A subset selection $\mathcal{Z}[6,7]$ is, by definition, a classtheoretic function sending each poset $P$ to a set $\mathcal{Z}(P)$ of subsets of $P$ whose elements are the socalled $\mathcal{Z}$-sets of $P$, and is called a subset system $[6,25]$ if it satisfies the additional property that for each monotone map $f: P \rightarrow Q, M \in \mathcal{Z}(P)$ implies $f(M) \in \mathcal{Z}(Q)$. It is said that a subset selection $\mathcal{Z}$ preserves surjectivity if for each surjective monotone map $f: P \rightarrow Q$ and for each $M \in \mathcal{Z}(Q)$, there exists at least one $N \in \mathcal{Z}(P)$ such that $M=f(N)$. Throughout this paper, $\mathcal{Z}$ and $\mathcal{Z}_{i}(i=1, \ldots, 4)$ always stand for subset systems if further assumptions are not made explicitly. In this paper, we consider only the subset systems $\mathcal{V}, \mathcal{F}, \mathcal{D}, \mathcal{C} n, \mathcal{P}$, where $\mathcal{V}$ $(\mathcal{F}, \mathcal{D}, \mathcal{C} n, \mathcal{P})$-sets of each poset $P$ are no subset (finite subsets, directed subsets, countable subsets and all subsets, resp.) of $P$. Note that all of them but $\mathcal{D}$ are surjectivity-preserving.

A poset $P$ is called $\mathcal{Z}-\bigvee(\bigwedge)$-complete iff each $M \in \mathcal{Z}(P)$ has a join (meet) in $P[4,7,25]$. A $\left(\mathcal{Z}_{1}, \mathcal{Z}_{2}\right)$-complete poset is defined to be a $\mathcal{Z}_{1}-\bigvee$ complete and $\mathcal{Z}_{2}$ - $\bigwedge$-complete poset [4]. If we associate two subset selections $\mathcal{Z}^{\text {sup }}$ and $\mathcal{Z}^{\text {inf }}$ to $\mathcal{Z}$ such that $M \in \mathcal{Z}^{\sup (\mathrm{inf})}(P)$ iff $M \in \mathcal{Z}(P)$ with $\bigvee M$ $(\bigwedge M)$, then $P$ is $\left(\mathcal{Z}_{1}, \mathcal{Z}_{2}\right)$-complete iff $\mathcal{Z}_{1}(P)=$ $\mathcal{Z}_{1}^{\text {sup }}(P)$ and $\mathcal{Z}_{2}(P)=\mathcal{Z}_{2}^{\text {inf }}(P)$.

A monotone function $f: P \rightarrow Q$ is $\mathcal{Z}$ - $\bigvee$ continuous iff for each $M \in \mathcal{Z}^{\text {sup }}(P), \bigvee f(M)=$ $f(\bigvee M)$, and is $\mathcal{Z}$-^-continuous iff for each $M \in \mathcal{Z}^{\text {inf }}(P), \bigwedge f(M)=f(\bigwedge M)$. A $\mathcal{Z}_{1^{-}}$ $\bigvee$-continuous and $\mathcal{Z}_{2}-\bigwedge$-continuous function is shortly called $\left(\mathcal{Z}_{1}, \mathcal{Z}_{2}\right)$-continuous function. For $\mathcal{Q}=\left(\mathcal{Z}_{1}, \mathcal{Z}_{2}, \mathcal{Z}_{3}, \mathcal{Z}_{4}\right),\left(\mathcal{Z}_{1}, \mathcal{Z}_{2}\right)$-complete posets and $\left(\mathcal{Z}_{3}, \mathcal{Z}_{4}\right)$-continuous maps constitute a category $\mathcal{Q P}$ [4]. For the sake of shortness and for any occurrence of $\mathcal{Q}=\left(\mathcal{Z}_{1}, \mathcal{Z}_{2}, \mathcal{Z}_{1}, \mathcal{Z}_{2}\right), \mathcal{Q}$ will be replaced by the pair $\left(\mathcal{Z}_{1}, \mathcal{Z}_{2}\right)$ in this paper, e.g. $\left(\mathcal{Z}_{1}, \mathcal{Z}_{2}, \mathcal{Z}_{1}, \mathcal{Z}_{2}\right) \mathbf{P}$ will be shortly written as $\left(\mathcal{Z}_{1}, \mathcal{Z}_{2}\right) \mathbf{P}$. As is shown in [4], most of the familiar order-theoretic constructs can be expressed in the form of $\mathcal{Q P}$. Now we give only some examples of $\mathcal{Q P}$ that will be used subsequently, and refer reader to [4] for many other examples.

Example 19. For $\mathcal{Q}=(\mathcal{V}, \mathcal{V}, \mathcal{V}, \mathcal{V}) \quad(\mathcal{Q}=$
$(\mathcal{F}, \mathcal{F}, \mathcal{F}, \mathcal{F}), \quad \mathcal{Q}=(\mathcal{P}, \mathcal{F}, \mathcal{P}, \mathcal{F}), \quad \mathcal{Q}=(\mathcal{V}, \quad \mathcal{P}$, $\mathcal{V}, \mathcal{P}), \quad \mathcal{Q}=(\mathcal{F}, \mathcal{P}, \mathcal{F}, \mathcal{P}), \quad \mathcal{Q}=(\mathcal{D}, \mathcal{P}, \mathcal{D}, \mathcal{P})$, $\mathcal{Q}=(\mathcal{P}, \mathcal{P}, \mathcal{P}, \mathcal{P}), \quad \mathcal{Q}=(\mathcal{C} n, \mathcal{F}, \mathcal{C} n, \mathcal{F}), \mathcal{Q}=$ $(\mathcal{D}, \mathcal{F}, \mathcal{D}, \mathcal{F})), \mathcal{Q P}$ is known as the category Pos of posets and monotone maps [1] (the category Blatt of bounded lattices and maps preserving finite joins and and finite meets [2], the category $\boldsymbol{S U} \boldsymbol{P}^{\wedge}$ of complete lattices and maps preserving arbitrary joins and finite meets [11], the category MCPos of complete lattices and maps preserving arbitrary meets [1], the category $\boldsymbol{I N}_{\mathbf{N}}{ }^{\vee}$ of complete lattices and maps preserving arbitrary meets and finite joins [4], the category $\boldsymbol{I N F}^{\uparrow}$ of complete lattices and maps preserving arbitrary meets and directed joins [11], the category CLat of complete lattices and maps preserving arbitrary meets and arbitrary joins [1], the category $\sigma$ ComLat of $\sigma$-complete lattices and maps preserving countable joins and finite meets [4], the category $\mathbf{Q F}$ of quasiframes and Scott-continuous functions preserving finite meets [9], resp.).

Since the functor $G_{\mathcal{Q}}: \mathcal{Q} \mathbf{P} \rightarrow \mathbf{P}$, defined by $G_{\mathcal{Q}}(P)=\left(P, \mathcal{Z}_{3}^{\sup }(P), \mathcal{Z}_{4}^{\inf }(P)\right)$ and $G_{\mathcal{Q}}(f)=f$, is a full embedding [4], $\mathbf{P}$ forms a category larger than $\mathcal{Q P}$. On the other hand, $\mathcal{Q P}$ can be viewed as the practically most realizable part of $\mathbf{P}$.

\subsection{Poset-valued $\mathcal{Q}$-spaces}

Definition 20. [4] The category $\mathcal{Q} \boldsymbol{S}$ consists of the following data: Objects are $\mathcal{Q}$-spaces $(X, \tau)$, that is, $X$ is a set and $\tau$ is a subset of $\mathcal{P}(X)$ with the property that $\tau$ is a $\left(\mathcal{Z}_{1}, \mathcal{Z}_{2}\right)$-complete poset ordered by set inclusion, and the inclusion map $i_{\tau}: \tau \hookrightarrow \mathcal{P}(X)$ is $\left(\mathcal{Z}_{3}, \mathcal{Z}_{4}\right)$-continuous. Morphisms $f:(X, \tau) \rightarrow(Y, \nu)$ are functions $f: X \rightarrow Y$ such that $\left(f^{\leftarrow}\right) \rightarrow(\nu) \subseteq \tau$.

As a natural poset-valued extension of $\mathcal{Q S}$, we now introduce, for an arbitrarily fixed $\left(\mathcal{Z}_{1}, \mathcal{Z}_{2}\right)$ complete poset $L$, the category $L$ - $\mathcal{Q S}$ of $L$ - $\mathcal{Q}$-spaces, and point out in this section that $L$ - $\mathcal{Q S}$ can be fully embedded into $G_{\mathcal{Q}}(L)-\mathbf{S}$. To present $L$ - $\mathcal{Q S}$, we should first note that by virtue of Proposition 1, each function $f: X \rightarrow Y$ gives rise to a $\mathcal{Q P}$ morphism $f_{L}^{\leftarrow}: L^{Y} \rightarrow L^{X}$, defined by $f_{L}^{\leftarrow}(\mu)=\mu \circ f$ for each $\mu \in L^{Y}$.

Definition 21. The category $L-\mathcal{Q} S$ comprises the following items: Objects are L-valued $\mathcal{Q}$-spaces $(X, \tau)$, i.e. $X$ is a set and $\tau$ is a subset of $L^{X}$ such that $\tau$ is a $\left(\mathcal{Z}_{1}, \mathcal{Z}_{2}\right)$-complete poset equipped with the order inherited from $L^{X}$, and the inclusion map $i_{\tau}: \tau \hookrightarrow L^{X}$ is $\left(\mathcal{Z}_{3}, \mathcal{Z}_{4}\right)$-continuous. Morphisms $f:(X, \tau) \rightarrow(Y, \nu)$ are functions $f: X \rightarrow Y$ such that $\left(f_{\overleftarrow{L}}^{\leftarrow}\right) \rightarrow(\nu) \subseteq \tau$.

In a similar way to Proposition 14, one can easily observe that $2-\mathcal{Q S}$ is isomorphic to $\mathcal{Q S}$. The category $L-\mathcal{Q S}$ enables us to unify the poset-valued extensions of the categories of various notions of spaces: 
Example 22. (1) For a poset $L$ and $\mathcal{Q}=$ $(\mathcal{V}, \mathcal{V}, \mathcal{V}, \mathcal{V}), L-\mathcal{Q} \boldsymbol{S}$, denoted by $L-\boldsymbol{B} \boldsymbol{S}$, is an extension of the category $\boldsymbol{B} \boldsymbol{S}$ of base spaces, extensively studied in [8].

(2) For a bounded lattice $L$ (i.e. $L$ is a lattice with the top and bottom elements) and $\mathcal{Q}=(\mathcal{F}, \mathcal{F}, \mathcal{F}, \mathcal{F})$, $L-\mathcal{Q S}$, denoted by $L-\boldsymbol{B l a t B S}$, is an extension of the full subcategory BlatBS of $\boldsymbol{B S}$ in [4].

(3) For a complete lattice $L$ and $\mathcal{Q}=$ $(\mathcal{P}, \mathcal{F}, \mathcal{P}, \mathcal{F}), L-\mathcal{Q} \boldsymbol{S}$, denoted by $L-\boldsymbol{T o p}$, is an extension of the category Top of topological spaces [1], and is also known as the category of L-topological spaces [20].

(4) For a complete lattice $L$ and $\mathcal{Q}=(\mathcal{V}, \mathcal{P}, \mathcal{V}, \mathcal{P})$, $L-\mathcal{Q}$, denoted by $L-\boldsymbol{C S p}$, is an extension of the category CSp of closure spaces [10].

(5) For a complete lattice $L$ and $\mathcal{Q}=$ $(\mathcal{F}, \mathcal{P}, \mathcal{F}, \mathcal{P}), L-\mathcal{Q} \boldsymbol{S}$, denoted by $L-\boldsymbol{T} \boldsymbol{C S p}$, is an extension of the category $\mathbf{T C S p}$ of topological closure spaces [10].

(6) For a complete lattice $L$ and $\mathcal{Q}=$ $(\mathcal{D}, \mathcal{P}, \mathcal{D}, \mathcal{P}), L-\mathcal{Q S}$, denoted by $L-\boldsymbol{A} \boldsymbol{C S p}$, is an extension of the category $\boldsymbol{A C S p}$ of algebraic closure spaces [10].

(7) For a complete lattice $L$ and $\mathcal{Q}=$ $(\mathcal{P}, \mathcal{P}, \mathcal{P}, \mathcal{P}), L-\mathcal{Q S}$, denoted by $L-\boldsymbol{A T S p}$, is an extension of the category ATSp of Alexandroffdiscrete spaces [10].

(8) For a $\sigma$-complete lattice $L$ (i.e. $L$ is a poset with countable joins and finite meets) and $\mathcal{Q}=$ $(\mathcal{C} n, \mathcal{F}, \mathcal{C} n, \mathcal{F}), L-\mathcal{Q} \boldsymbol{S}$, denoted by $L$-Alex, is an extension of the category Alex of Alexandroff spaces [2, 4].

(9) For a quasiframe L [9] (i.e. $L$ is a poset with directed joins and finite meets ) and $\mathcal{Q}=$ $(\mathcal{D}, \mathcal{F}, \mathcal{D}, \mathcal{F}), L-\mathcal{Q S}$, denoted by L-PreTop, is an extension of the category PreTop of pretopological spaces [9].

It is shown in [4] that the functor $H_{\mathcal{Q}}: \mathcal{Q S} \rightarrow \mathbf{S}$, defined by $H_{\mathcal{Q}}(X, \tau)=\left(X, \tau, \mathcal{Z}_{3}^{\text {sup }}(\tau), \mathcal{Z}_{4}^{\inf }(\tau)\right)$ and $H_{\mathcal{Q}}(f)=f$, is a full embedding. We now extend this result to $L$ - $\mathcal{Q}$-spaces.

Lemma 23. Let $X$ be a set, and let $\tau$ be a subset of $L^{X}$ such that $\tau$ is a $\left(\mathcal{Z}_{1}, \mathcal{Z}_{2}\right)$-complete poset equipped with the order inherited from $L^{X}$. Then, $(X, \tau)$ is an $L$-Q -space iff $L H_{\mathcal{Q}}(X, \tau)=$ $\left(X, \tau, \mathcal{Z}_{3}^{\sup }(\tau), \mathcal{Z}_{4}^{\inf }(\tau)\right)$ is a $G_{\mathcal{Q}}(L)$-space.

Proof. Let $(X, \tau)$ be an $L$ - $\mathcal{Q}$-space. $G_{\mathcal{Q}}(L) T\left(L H_{\mathcal{Q}}(X, \tau)\right)$ is obviously an augmented poset. On the other hand, since $i_{\tau}: \tau \hookrightarrow L^{X}$ is a $\mathcal{Q} \mathbf{P}$-morphism, $G_{\mathcal{Q}}\left(i_{\tau}\right): G_{\mathcal{Q}}(\tau) \rightarrow G_{\mathcal{Q}}\left(L^{X}\right)$ is a $\mathbf{P}$-morphism. Furthermore, we easily see that the identity map $i d_{L^{X}}$ on $L^{X}$ is a P-morphism $G_{\mathcal{Q}}\left(L^{X}\right) \rightarrow G_{\mathcal{Q}}(L)^{X}$. Thus $i_{\tau}=i d_{L^{X}} \circ G_{\mathcal{Q}}\left(i_{\tau}\right): G_{\mathcal{Q}}(\tau) \rightarrow G_{\mathcal{Q}}(L)^{X}$ is a $\mathbf{P}$-morphism. Then it follows from Remark 12 (i) that $L H_{\mathcal{Q}}(X, \tau)$ is a $G_{\mathcal{Q}}(L)$-space. Conversely, suppose $L H_{\mathcal{Q}}(X, \tau)$ is a $G_{\mathcal{Q}}(L)$-space. Then, $i_{\tau}: G_{\mathcal{Q}}(\tau) \rightarrow G_{\mathcal{Q}}(L)^{X}$ is a $\mathbf{P}$-morphism by Remark 12 (i). To see that $(X, \tau)$ is an $L$ - $\mathcal{Q}$-space, it is enough to confirm that the inclusion map $i_{\tau}: \tau \hookrightarrow L^{X}$ is $\left(\mathcal{Z}_{3}, \mathcal{Z}_{4}\right)$-continuous. For each $M \in \mathcal{Z}_{3}^{\sup }(\tau)$, since $i_{\tau}: G_{\mathcal{Q}}(\tau) \rightarrow G_{\mathcal{Q}}(L)^{X}$ is a $\mathbf{P}$-morphism, we have $i_{\tau}(\bigvee M)=\bigvee i_{\tau}(M)$, i.e. $i_{\tau}$ is $\mathcal{Z}_{3}-\bigvee$-continuous. $\mathcal{Z}_{4}-\bigwedge$-continuity of $i_{\tau}$ is similar.

Theorem 24. The functor $L H_{\mathcal{Q}}: L-\mathcal{Q S} \rightarrow$ $G_{\mathcal{Q}}(L)-S$, sending each $(X, \tau)$ to $L H_{\mathcal{Q}}(X, \tau)$, and leaving morphisms unchanged, is a full embedding.

Proof. We have from Lemma 23 that $L H_{\mathcal{Q}}$ assigns

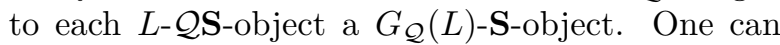
also prove that each $L$-QS-morphism $(X, \tau) \stackrel{f}{\rightarrow}$ $(Y, \nu)$ yields a $G_{\mathcal{Q}}(L)$-S-morphism $L H_{\mathcal{Q}}(X, \tau) \stackrel{f}{\rightarrow}$ $L H_{\mathcal{Q}}(Y, \nu)$. Because $L H_{\mathcal{Q}}$ clearly preserves composition and the identities, $L H_{\mathcal{Q}}: L$ - $\mathcal{Q S} \rightarrow G_{\mathcal{Q}}(L)-\mathbf{S}$ will be, indeed, a functor. The property of $L H_{\mathcal{Q}}$ being a full embedding is easily seen from the definition of $L H_{\mathcal{Q}}$.

Theorem 24 proves that $A$-spaces provide a more general approach than $L$ - $\mathcal{Q}$-spaces. However, as is exhibited in Example 22, $L$ - $\mathcal{Q}$-spaces produce more efficient and easily realizable results.

\subsection{Relations between $L-\mathcal{Q S}$ and $\mathcal{Q} \mathbf{P}^{o p}$}

In this section, by providing suitable definitions of $L$ - $\mathcal{Q}$-spatiality in $\mathcal{Q P}$ and $L$ - $\mathcal{Q}$-sobriety in $L$ - $\mathcal{Q S}$, our aim is to show that $A T \dashv A \Psi: \mathbf{P}^{o p} \rightarrow A-\mathbf{S}$ and $A$-SpaP ${ }^{o p} \sim A$-SobS can be carried over an adjunction between $L$ - $\mathcal{Q S}$ and the opposite of the full subcategory $L-\mathcal{Q} \mathbf{P}_{s}$ of $\mathcal{Q P}$ of all $L$ - $\mathcal{Q}$-spatial objects and a dual equivalence between $L-\mathcal{Q} \mathbf{P}_{s}$ and the full subcategory of $L$ - $\mathcal{Q S}$ of all $L$ - $\mathcal{Q}$-sober objects, respectively. In case $\mathcal{Z}_{1}$ and $\mathcal{Z}_{2}$ preserve surjectivity, we shall also demonstrate that $A T \dashv A \Psi$ : $\mathbf{P}^{o p} \rightarrow A-\mathbf{S}$ produces an adjunction between $L$ $\left(\mathcal{Z}_{1}, \mathcal{Z}_{2}\right) \mathbf{S}$ and $\left(\mathcal{Z}_{1}, \mathcal{Z}_{2}\right) \mathbf{P}^{o p}$. We start with some preparations.

Proposition 25. [4] For categories $\boldsymbol{A}, \boldsymbol{B}$, a full subcategory $\boldsymbol{A}^{\prime}$ of $\boldsymbol{A}$ and a full subcategory $\boldsymbol{B}^{\prime}$ of $\boldsymbol{B}$, if $F \dashv G: \boldsymbol{A} \rightarrow \boldsymbol{B}$ is an adjoint pair of functors with the property that for all $X \in O b\left(\mathbf{A}^{\prime}\right)$ and for all $Y \in O b\left(\mathbf{B}^{\prime}\right)$,

$$
G(X) \in O b\left(\mathbf{B}^{\prime}\right) \text { and } F(Y) \in O b\left(\mathbf{A}^{\prime}\right)
$$

then the restriction $F^{\prime}$ of $F$ to $\boldsymbol{B}^{\prime}$ and the restriction $G^{\prime}$ of $G$ to $\boldsymbol{A}^{\prime}$ form an adjoint pair of functors $F^{\prime} \dashv$ $G^{\prime}: \boldsymbol{A}^{\prime} \rightarrow \boldsymbol{B}^{\prime}$.

Corollary 26. [4] Under the considerations in Proposition 25, if $G: \boldsymbol{A} \rightarrow \boldsymbol{B}$ and $F: B \rightarrow \boldsymbol{A}$ are equivalences inverse to each other and satisfy (1), then $G^{\prime}: \boldsymbol{A}^{\prime} \rightarrow \boldsymbol{B}^{\prime}$ and $F^{\prime}: \boldsymbol{B}^{\prime} \rightarrow \boldsymbol{A}^{\prime}$ are equivalences inverse to each other. 
Lemma 27. Let $\boldsymbol{P}(\mathcal{Q})$ and $\boldsymbol{S}(L, \mathcal{Q})$ stand for the image of $\mathcal{Q P}$ under $G_{\mathcal{Q}}$ and the image of $L-\mathcal{Q S}$ under $L H_{\mathcal{Q}}$, respectively. Then, the functor $G_{\mathcal{Q}}(L) T$ : $G_{\mathcal{Q}}(L)-\boldsymbol{S} \rightarrow \boldsymbol{P}^{o p}$ assigns to each $\boldsymbol{S}(L, \mathcal{Q})$-object a $\boldsymbol{P}(\mathcal{Q})$-object.

Proof. Since each $\mathbf{S}(L, \mathcal{Q})$-object $W$ possesses the property that $\mathfrak{O}_{G_{\mathcal{Q}}(L)}(W)$ is a $\left(\mathcal{Z}_{1}, \mathcal{Z}_{2}\right)$-complete poset and $G_{\mathcal{Q}}(L) T(W)=G_{\mathcal{Q}}\left(\mathfrak{O}_{G_{\mathcal{Q}}(L)}(W)\right)$, it is clear from the definition of $\mathbf{P}(\mathcal{Q})$ that $G_{\mathcal{Q}}(L) T(W)$ is a $\mathbf{P}(\mathcal{Q})$-object.

Lemma 28. Let $\mathcal{Z}_{1}$ and $\mathcal{Z}_{2}$ preserve surjectivity. Then, the functor $G_{\left(\mathcal{Z}_{1}, \mathcal{Z}_{2}\right)}(L) \Psi$ maps each $\boldsymbol{P}\left(\mathcal{Z}_{1}, \mathcal{Z}_{2}\right)$-object to an $\boldsymbol{S}\left(L, \mathcal{Z}_{1}, \mathcal{Z}_{2}\right)$-object.

Proof. The proof can be done by using analogous arguments to those in the proof of [4, Lemma 6], so it is omitted here.

Lemma 29. Let $\boldsymbol{P}(L, \mathcal{Q})_{s}$ denote the full subcategory of $\boldsymbol{P}(\mathcal{Q})$ of all $G_{\mathcal{Q}}(L)$-spatial objects. Then $G_{\mathcal{Q}}(L) \Psi$ maps each $\boldsymbol{P}(L, \mathcal{Q})_{s}$-object to an $\boldsymbol{S}(L, \mathcal{Q})$ object.

Proof. The required result can be verified in a similar fashion to [4, Lemma 8].

Definition 30. Let $P$ be a $\left(\mathcal{Z}_{1}, \mathcal{Z}_{2}\right)$-complete poset, and $(X, \tau)$ an $L$ - $\mathcal{Q}$-space.

(i) $P$ is $L$-Q $\mathcal{Q}$-spatial iff $G_{\mathcal{Q}}(P)$ is $G_{\mathcal{Q}}(L)$-spatial.

(ii) $(X, \tau)$ is $L$ - $\mathcal{Q}$-sober iff $L H_{\mathcal{Q}}(X, \tau)$ is $G_{\mathcal{Q}}(L)$ sober.

Theorem 31. Let $L-\mathcal{Q} \boldsymbol{P}_{s}$ be the full subcategory

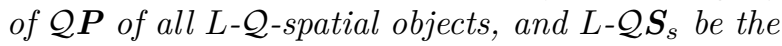
full subcategory of $L-\mathcal{Q} S$ of all $L$ - $\mathcal{Q}$-sober objects. Then the following statements are true:

(i) There is a pair of adjoint functors $L T_{\mathcal{Q}} \dashv$ $L \Psi_{\mathcal{Q}}: L-\mathcal{Q} \boldsymbol{P}_{s}^{o p} \rightarrow L-\mathcal{Q} S$.

(ii) $L-\mathcal{Q} \boldsymbol{P}_{s}$ is dually equivalent to $L-\mathcal{Q} \boldsymbol{S}_{s}$.

(iii) If $\mathcal{Z}_{1}$ and $\mathcal{Z}_{2}$ are surjectivity-preserving, then there is a pair of adjoint functors $L T_{\left(\mathcal{Z}_{1}, \mathcal{Z}_{2}\right)} \dashv$ $L \Psi_{\left(\mathcal{Z}_{1}, \mathcal{Z}_{2}\right)}:\left(\mathcal{Z}_{1}, \mathcal{Z}_{2}\right) \boldsymbol{P}^{o p} \rightarrow L-\left(\mathcal{Z}_{1}, \mathcal{Z}_{2}\right) S$.

Proof. (i) To prove the assertion, we apply Proposition 25, and choose $F \dashv G: \mathbf{A} \rightarrow \mathbf{B}$ as $G_{\mathcal{Q}}(L) T \dashv$ $G_{\mathcal{Q}}(L) \Psi: \mathbf{P}^{o p} \rightarrow G_{\mathcal{Q}}(L)$-S. By considering the fact that the co-domain of $G_{\mathcal{Q}}(L) T$ lies in $\mathbf{P}(L, \mathcal{Q})_{s}^{o p}$, and using Lemma 27 and Lemma 29, we observe that the condition (1) in Proposition 25 is satisfied for $\mathbf{A}^{\prime}=\mathbf{P}(L, \mathcal{Q})_{s}^{o p}$ and $\mathbf{B}^{\prime}=\mathbf{S}(L, \mathcal{Q})$. Thus the adjunction in question follows from Proposition 25 and the fact that $\mathbf{P}(L, \mathcal{Q})_{s}$ and $\mathbf{S}(L, \mathcal{Q})$ are, respectively, isomorphic to $L-\mathcal{Q P} \mathbf{P}_{s}$ and $L-\mathcal{Q S}$.

(ii) is an application of Corollary 26 to the equivalences $G_{\mathcal{Q}}(L) \Psi_{s}: G_{\mathcal{Q}}(L)$-SpaP ${ }^{o p} \rightarrow G_{\mathcal{Q}}(L)$ SobS and $G_{\mathcal{Q}}(L) T_{s}: G_{\mathcal{Q}}(L)$-SobS $\rightarrow G_{\mathcal{Q}}(L)$ SpaP $^{o p}$. By making use of the isomorphism between $\mathbf{P}(L, \mathcal{Q})_{s}$ and $L-\mathcal{Q} \mathbf{P}_{s}$, the full subcategory of $\mathbf{S}(L, \mathcal{Q})$ of all $G_{\mathcal{Q}}(L)$-sober spaces and $L-\mathcal{Q} \mathbf{S}_{s}$, the required equivalence follows from Lemma 27 and Lemma 29. (iii) Firstly, pick $F \dashv G: \mathbf{A} \rightarrow \mathbf{B}$ as $G_{\left(\mathcal{Z}_{1}, \mathcal{Z}_{2}\right)}(L) T \dashv G_{\left(\mathcal{Z}_{1}, \mathcal{Z}_{2}\right)}(L) \Psi: \mathbf{P}^{o p} \rightarrow G_{\left(\mathcal{Z}_{1}, \mathcal{Z}_{2}\right)}(L)-$ $\mathbf{S}, \quad \mathbf{A}^{\prime}=\mathbf{P}\left(\mathcal{Z}_{1}, \mathcal{Z}_{2}\right)^{o p}$ and $\mathbf{B}^{\prime}=\mathbf{S}\left(L, \mathcal{Z}_{1}, \mathcal{Z}_{2}\right)$ in Proposition 25. Then, since $\left(\mathcal{Z}_{1}, \mathcal{Z}_{2}\right) \mathbf{P}$ and $L$ $\left(\mathcal{Z}_{1}, \mathcal{Z}_{2}\right) \mathbf{S}$ are, respectively, isomorphic to $\mathbf{P}\left(\mathcal{Z}_{1}, \mathcal{Z}_{2}\right)$ and $\mathbf{S}\left(L, \mathcal{Z}_{1}, \mathcal{Z}_{2}\right)$, we obtain the questioned pair of adjoint functors from Proposition 25 by making use of Lemma 27 and Lemma 28.

Theorem 31 is an extension of the main result of [4] ([4, Theorem 2]) to $L$ - $\mathcal{Q}$-spaces, and has direct applications to many familiar categories of orderedstructures. We gather some (but not all) of them in the final result:

Corollary 32. Let $L$ be an object of Pos (Blatt, SUP ${ }^{\wedge}, M C P o s, I N F^{\vee}, I N F^{\uparrow}, C L a t, \sigma C o m L a t$ and $\boldsymbol{Q F})$. Then, there are adjunctions between $L$ BS and Pos $^{o p}, L-B l a t B S$ and Blatt ${ }^{o p}, L-$ Top and $\left(\boldsymbol{S U P} \boldsymbol{P}^{\wedge}\right)^{o p}, L-C S p$ and $\mathbf{M C P o s}^{o p}, L-\boldsymbol{T C S p}$ and $\left(\boldsymbol{I N} \boldsymbol{F}^{\vee}\right)^{o p}, L-\boldsymbol{A C S p}$ and $\left(L-\boldsymbol{I N} \boldsymbol{F}_{s}^{\uparrow}\right)^{o p}, L-\boldsymbol{A T S} \boldsymbol{p}$ and CLat $^{o p}, L$-Alex and $\sigma$ ComLat ${ }^{o p}, L$-PreTop and $\left(L-\boldsymbol{Q} \boldsymbol{F}_{s}\right)^{o p}$. Furthermore, there are dual equivalences between $L-\boldsymbol{P o s}_{s}$ and $L-\boldsymbol{B} \boldsymbol{S}_{s}, L-\boldsymbol{B l a t t}_{s}$ and $L-$ BlatBS $_{s}, L-S U \boldsymbol{P}_{s}^{\wedge}$ and $L-\boldsymbol{T o p}_{s}, L-\mathrm{MCPos}_{s}$

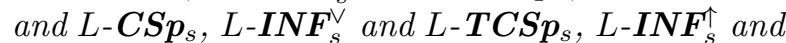
$L-\boldsymbol{A C S p} \boldsymbol{p}_{s}, L-\boldsymbol{C L a t} t_{s}$ and $L-\boldsymbol{A T S p _ { s }}, L-\sigma$ ComLat $_{s}$ and $L-\boldsymbol{A l e x}_{s}, L-\boldsymbol{Q F}{ }_{s}$ and $L-\boldsymbol{P r e T o p _ { s }}$.

\section{References}

[1] J. Adámek, H. Herrlich and G. E. Strecker, Abstract and Concrete Categories, Wiley, New York, 1990.

[2] B. Banaschewski and G. Bruns, The fundamental duality of partially ordered sets, Order, 5:61-74, 1988.

[3] M. Demirci, Pointed semi-quantales and lattice-valued topological spaces, Fuzzy Sets and Systems, 161:1224-1241, 2010.

[4] M. Demirci, $\left(\mathcal{Z}_{1}, \mathcal{Z}_{2}\right)$-complete partially ordered sets and their representations by $\mathcal{Q}$-spaces, Appl. Categ. Struct., doi: 10.1007/s10485-012-9277-4.

[5] M. Demirci, Fundamental duality of abstract categories and its applications, Fuzzy Sets and Systems, Submitted.

[6] M. Erné, Bigeneration in complete lattices and principle separation in posets, Order, 8:197221, 1991.

[7] M. Erné, Algebraic ordered sets and their generalizations. In I. Rosenberg and G. Sabidussi, editors, Algebras and Orders, Proc. Montreal, 1992, pages 113-192, Kluwer Academic Publishers, Amsterdam, 1993.

[8] M. Erné, General Stone duality, Topol. its Appl., 137:125-158, 2004.

[9] M. Erné, Choiceless, pointless, but not useless: dualities for preframes, Appl. Categ. Struct., 15:541-572, 2007. 
[10] M. Erné, Closure. In F. Mynard et al., editors, Beyond topology, Contemporary Mathematics 486, pages 163-238. American Mathematical Society, Providence, 2009.

[11] G. Gierz, K. H. Hofmann, K. Keimel, J. D. Lawson, M. Mislove and D. S. Scott, Continuous Lattices and Domains, Cambridge University Press, Cambridge, 2003.

[12] J. A. Goguen, Categories of V-sets, Bull. Amer. Math. Soc., 75:622-624, 1969.

[13] H. Herrlich and G. E. Strecker, Category Theory, Allyn Bacon, Boston, 1973.

[14] U. Höhle, Many valued topology and its applications, Kluwer Academic Publishers, Boston, 2001.

[15] U. Höhle and A.P. Šostak, Axiomatic foundations of fixed-basis fuzzy topology. In U. Höhle and S. E. Rodabaugh, editors, Mathematics of Fuzzy Sets: Logic, Topology and Measure Theory, Handbook of Fuzzy Sets Series, Vol. 3, pages 123272, Kluwer Academic Publishers, Dordrecht, 1999.

[16] J. R. Isbell, Atomless parts of spaces, Math. Scand., 31:5-32, 1972.

[17] P. T. Johnstone, Stone Spaces, Cambridge University Press, 1982.

[18] S. Mac Lane, Categories for the Working Mathematician, Springer, Berlin, 1971.

[19] D. Papert, S. Papert, Sur les treillis des ouverts et les paratopologies, Semin. de Topologie et de Geometrie Differentielle Ch. Ehresmann 1: 1-9, 1959.

[20] S. E. Rodabaugh, Point-set lattice-theoretic topology, Fuzzy Sets and Systems 40: 297-345, 1991.

[21] S. E. Rodabaugh, Powerset operator foundations for poslat fuzzy set theories and topologies. In U. Höhle and S. E. Rodabaugh, editors, Mathematics of Fuzzy Sets: Logic, Topology and Measure Theory, Handbook of Fuzzy Sets Series, Vol. 3, pages 91-116, Kluwer Academic Publishers, Dordrecht, 1999.

[22] S. E. Rodabaugh, Relationship of algebraic theories to powerset theories and fuzzy topological theories for lattice-valued mathematics, Int. J. Math. Math. Sci., 2007:1-71, 2007.

[23] S. A. Solovyov, Sobriety and spatiality in varieties of algebras, Fuzzy Sets Systems, 159:25672585, 2008.

[24] W. Yao, A survey of fuzzifications of frames, the Papert-Papert-Isbell adjunction and sobriety, 190:63-81, 2012.

[25] J. B. Wright, E. G. Wagner and J. W. Thatcher, A uniform approach to inductive posets and inductive closure, Theoret. Comput. Sci., 7:57-77, 1978.

[26] L. A. Zadeh, Fuzzy sets, Inform. and Control, 8:338-353, 1965. 